\title{
HOMOGENEOUS AND $H$-CONTACT UNIT TANGENT SPHERE BUNDLES
}

\author{
G. CALVARUSO and D. PERRONE \\ (Received 2 September 2009; accepted 1 February 2010) \\ Communicated by M. K. Murray
}

\begin{abstract}
We prove that all $g$-natural contact metric structures on a two-point homogeneous space are homogeneous contact. The converse is also proved for metrics of Kaluza-Klein type. We also show that if $(M, g)$ is an Einstein manifold and $\tilde{G}$ is a Riemannian $g$-natural metric on $T_{1} M$ of Kaluza-Klein type, then $\left(T_{1} M, \tilde{\eta}, \tilde{G}\right)$ is $H$-contact if and only if $(M, g)$ is 2 -stein, so proving that the main result of Chun et al. [' $H$-contact unit tangent sphere bundles of Einstein manifolds', $Q$. J. Math., to appear. DOI: 10.1093/qmath/hap025] is invariant under a two-parameter deformation of the standard contact metric structure on $T_{1} M$. Moreover, we completely characterize Riemannian manifolds admitting two distinct $H$-contact $g$-natural contact metric structures, with associated metric of Kaluza-Klein type.
\end{abstract}

2000 Mathematics subject classification: primary 53C15; secondary 53C25, 53D10.

Keywords and phrases: two-point homogeneous spaces, unit tangent sphere bundle, $g$-natural metric, $H$-contact spaces.

\section{Introduction}

The study of the relationships between the geometric properties of a Riemannian manifold $M$ and those of its unit tangent sphere bundle $T_{1} M$ is a well-known and interesting research field in Riemannian geometry. Usually, the properties of $T_{1} M$ influence those of the base manifold $M$ itself, and conversely. In particular, several authors have tried to characterize two-point homogeneous spaces via some conditions on the unit tangent sphere bundle.

It is well known [25] that a connected, simply connected two-point homogeneous space is either flat or isometric to a rank-one symmetric space (either $\mathbb{R} \mathbb{P}^{n}, \mathbb{S}^{n}, \mathbb{C} \mathbb{P}^{n}$, $\mathbb{H} \mathbb{P}^{n}, \mathbb{C}$ ay $\mathbb{P}^{2}$ or one of their noncompact duals).

The geometry of the unit tangent sphere bundle $T_{1} M$ is strongly influenced by the fact that the base manifold $(M, g)$ is two-point homogeneous. The Sasaki metric $g_{S}$ is the simplest and most natural Riemannian metric that can be considered on the tangent and unit tangent sphere bundles of a Riemannian manifold. With respect to this metric,

The authors are supported by funds of the University of Salento and the MIUR (PRIN 2007).

(C) 2010 Australian Mathematical Publishing Association Inc. 1446-7887/2010 \$16.00 
and to the homothetic metric $(1 / 4) g_{S}$ of the standard contact metric structure of $T_{1} M$, the following results hold.

(i) If $(M, g)$ is two-point homogeneous, then $\left(T_{1} M, g_{S}\right)$ is locally homogeneous $[20,25]$.

(ii) If $(M, g)$ is two-point homogeneous, then the standard contact metric structure of $T_{1} M$ is $H$-contact (equivalently, the geodesic flow vector field of $\left(T_{1} M, g_{S}\right)$ is harmonic) [12].

(iii) $(M, g)$ is globally Osserman if and only if the standard contact metric structure of $T_{1} M$ is locally homogeneous [10].

To our knowledge, the questions whether the converse of result (i) holds is still open. Some partial positive answers for the converse in (i) and (ii) were given in $[12,13]$. Very recently a characterization was obtained for Einstein spaces $(M, g)$ whose unit tangent sphere bundle is $H$-contact. These spaces must be 2 -stein, and this fact allows us to find plenty of examples of Riemannian manifolds that are not two-point homogeneous but have an $H$-contact $T_{1} M$ [17]. Because of these results, the following problems arise naturally.

Question 1.1 [11]. If $T_{1} M$ is homogeneous, is $(M, g)$ necessarily two-point homogeneous?

Question 1.2 [17]. If $T_{1} M$ is $H$-contact, is $(M, g)$ Einstein?

Questions 1.1 and 1.2 referred in [11] to the Sasaki metric and in [17] to the standard contact metric structure on $T_{1} M$, respectively. However, they also make sense for more general Riemannian metrics and contact metric structures.

In recent years, a very large family of metrics on the tangent bundle $T M$, called $g$-natural metrics, has been introduced and studied [7]. This family of metrics includes $g_{S}$ and, more generally, all Kaluza-Klein metrics, which are also relevant for applications to physics. Riemannian $g$-natural metrics on $T M$ depend on six arbitrary smooth real functions. Their restrictions to the hypersurface $T_{1} M$ are again called $g$-natural. They possess a simpler form but still depend on four arbitrary real parameters, satisfying some inequalities [6].

In [1], the first author and Abbassi replaced the standard contact metric structure of $T_{1} M$ by a family of contact metric structures $(\tilde{\eta}, \tilde{G})$, called $g$-natural contact metric structures. The Riemannian metrics $\tilde{G}$ of these contact structures are $g$-natural, and the characteristic vector field is collinear to the geodesic flow vector field. The relations between the contact metric geometry of $\left(T_{1} M, \tilde{\eta}, \tilde{G}\right)$ and the geometry of the base manifold were studied in $[1,3]$, and several properties turned out to be related (via the Osserman conjecture) to the base manifold being two-point homogeneous. The harmonicity of the geodesic flow vector field of the unit tangent sphere bundle of a two-point homogeneous space, with respect to arbitrary Riemannian $g$-natural metrics, was investigated by the present authors and Abbassi [4].

Finally, Kowalski and Sekizawa [19] showed the invariance of any $g$-natural metric on $T M$ with respect to the induced map of a (local) isometry of $(M, g)$. Using this fact, they extended result (i) above to all $g$-natural metrics, proving the following theorem. 
THEOREM 1.3 [19]. The tangent sphere bundle $T_{r} M$ of any radius $r>0$ of a twopoint homogeneous space, equipped with any Riemannian g-natural metric, is locally homogeneous.

In this paper, we study Questions 1.1 and 1.2 above, equipping the unit tangent sphere bundle $T_{1} M$ with some Riemannian $g$-natural metrics to which we shall refer as metrics of Kaluza-Klein type (see Section 3). This class of $g$-natural metrics includes the Kaluza-Klein metrics (in particular, both $g_{S}$ and the Cheeger-Gromoll metric) and is defined by a clear geometrical condition: it is formed by Riemannian $g$-natural metrics for which the horizontal and tangential distributions are mutually orthogonal. Investigating metrics of Kaluza-Klein type and associated contact metric structures on $T_{1} M$, we shall obtain some new characterizations of two-point homogeneous and $H$-contact spaces in terms of geometric properties of the unit tangent sphere bundle.

The paper is organized as follows. In Section 2 we recall the definition and basic properties of $g$-natural metrics. We describe $g$-natural contact metric structures on $T_{1} M$ in Section 3, where we prove that if the base manifold is two-point homogeneous, then such structures are homogeneous contact (Theorem 3.1). In Section 4, we answer Question 1.1 for metrics of Kaluza-Klein type. More precisely, we prove that if $(M, g)$ is a Riemannian manifold of dimension $n \neq 16$ and $\tilde{G}$ is an arbitrary $g$-natural metric on $T_{1} M$ of Kaluza-Klein type, then $(M, g)$ is (locally isometric to) a two-point homogeneous space if and only if $\left(T_{1} M, \tilde{G}\right)$ is (locally) homogeneous and the geodesic flow is invariant under the (local) isometries acting transitively on $T_{1} M$ (Theorems 4.2 and 4.3). Finally, in Section 5, we prove that if $(M, g)$ is an Einstein manifold and $\tilde{G}$ is a Riemannian $g$-natural metric on $T_{1} M$ of Kaluza-Klein type, then $\left(T_{1} M, \tilde{\eta}, \tilde{G}\right)$ is $H$-contact if and only if $(M, g)$ is 2-stein (Theorem 5.2). Consequently, the main result of [17] is invariant under a twoparameter deformation of the standard contact metric structure on $T_{1} M$. Moreover, with regard to Question 1.2, we completely characterize Riemannian manifolds admitting two distinct $H$-contact $g$-natural contact metric structures, whose associated metric is of Kaluza-Klein type (Theorem 5.3).

\section{Preliminaries on $g$-natural metrics}

Let $(M, g)$ be an $n$-dimensional Riemannian manifold and $\nabla$ be its Levi-Civita connection. At any point $(x, u)$ of its tangent bundle $T M$, the tangent space of $T M$ splits into the horizontal and vertical subspaces with respect to $\nabla$ :

$$
(T M)_{(x, u)}=\mathcal{H}_{(x, u)} \oplus \mathcal{V}_{(x, u)}
$$

For any vector $X \in M_{x}$, there exists a unique vector $X^{h} \in \mathcal{H}_{(x, u)}$ (the horizontal lift of $X$ to $(x, u) \in T M)$, such that $\pi_{*} X^{h}=X$, where $\pi: T M \rightarrow M$ is the natural projection. The vertical lift of a vector $X \in M_{x}$ to $(x, u) \in T M$ is a vector $X^{v} \in \mathcal{V}_{(x, u)}$ such that $X^{v}(d f)=X f$, for all functions $f$ on $M$. Here we consider 1-forms $d f$ on $M$ as functions on $T M$ (that is, $(d f)(x, u)=u f$ ). The map $X \rightarrow X^{h}$ is an 
isomorphism between the vector spaces $M_{x}$ and $\mathcal{H}_{(x, u)}$. Similarly, the map $X \rightarrow X^{v}$ is an isomorphism between $M_{x}$ and $\mathcal{V}_{(x, u)}$. Horizontal and vertical lifts of vector fields on $M$ can be defined in an obvious way and are uniquely defined vector fields on $T M$.

Riemannian g-natural metrics form a wide family of Riemannian metrics on TM. These metrics depend on several smooth functions from $\mathbb{R}^{+}=[0,+\infty)$ to $\mathbb{R}$ and, as their name suggests, they arise from a very 'natural' construction starting from a Riemannian metric $g$ over $M$. In fact, $g$-natural metrics are the image of $g$ under firstorder natural operators $D: S_{+}^{2} T^{*} \rightsquigarrow\left(S^{2} T^{*}\right) T$, which transform Riemannian metrics on manifolds into metrics on their tangent bundles, where $S_{+}^{2} T^{*}$ and $S^{2} T^{*}$ denote the bundle functors of all Riemannian metrics and all symmetric $(0,2)$-tensors over $n$-manifolds, respectively.

Given an arbitrary $g$-natural metric $G$ on the tangent bundle $T M$ of a Riemannian manifold $(M, g)$, there exist smooth functions $\alpha_{i}, \beta_{i}: \mathbb{R}^{+} \rightarrow \mathbb{R}$, where $i=1,2,3$, such that

$$
\begin{gathered}
G_{(x, u)}\left(X^{h}, Y^{h}\right)=\left(\alpha_{1}+\alpha_{3}\right)\left(r^{2}\right) g_{x}(X, Y) \\
\quad+\left(\beta_{1}+\beta_{3}\right)\left(r^{2}\right) g_{x}(X, u) g_{x}(Y, u), \\
\begin{aligned}
G_{(x, u)}\left(X^{h}, Y^{v}\right)= & G_{(x, u)}\left(X^{v}, Y^{h}\right)=\alpha_{2}\left(r^{2}\right) g_{x}(X, Y) \\
& \quad+\beta_{2}\left(r^{2}\right) g_{x}(X, u) g_{x}(Y, u), \\
G_{(x, u)}\left(X^{v}, Y^{v}\right)= & \alpha_{1}\left(r^{2}\right) g_{x}(X, Y)+\beta_{1}\left(r^{2}\right) g_{x}(X, u) g_{x}(Y, u),
\end{aligned}
\end{gathered}
$$

for every $u, X, Y \in M_{x}$, where $r^{2}=g_{x}(u, u)$. Put

$$
\begin{gathered}
\phi_{i}(t)=\alpha_{i}(t)+t \beta_{i}(t), \\
\alpha(t)=\alpha_{1}(t)\left(\alpha_{1}+\alpha_{3}\right)(t)-\alpha_{2}^{2}(t), \\
\phi(t)=\phi_{1}(t)\left(\phi_{1}+\phi_{3}\right)(t)-\phi_{2}^{2}(t),
\end{gathered}
$$

for all $t \in \mathbb{R}^{+}$. Then, a $g$-natural metric $G$ on $T M$ is Riemannian if and only if the following inequalities hold:

$$
\alpha_{1}(t)>0, \quad \phi_{1}(t)>0, \quad \alpha(t)>0, \quad \phi(t)>0,
$$

for all $t \in \mathbb{R}^{+}$.

In literature, there are some well-known Riemannian metrics on the tangent sphere bundle, which turn out to be special cases of Riemannian $g$-natural metrics (satisfying (2.2)). In particular:

(i) the Sasaki metric $g_{S}$ is obtained for

$$
\alpha_{1}(t)=1, \quad \alpha_{2}(t)=\alpha_{3}(t)=\beta_{1}(t)=\beta_{2}(t)=\beta_{3}(t)=0 ;
$$

(ii) the Cheeger-Gromoll metric $g_{G C}[15]$ is obtained when

$$
\alpha_{2}(t)=\beta_{2}(t)=0, \quad \alpha_{1}(t)=\beta_{1}(t)=-\beta_{3}(t)=\frac{1}{1+t}, \quad \alpha_{3}(t)=\frac{t}{1+t} ;
$$


(iii) Kaluza-Klein metrics, as commonly defined on principal bundles [8], are obtained for

$$
\alpha_{2}(t)=\beta_{2}(t)=\beta_{1}(t)+\beta_{3}(t)=0 .
$$

Notice that all metrics above satisfy $\alpha_{2}=\beta_{2}=0$, so they are $g$-natural Riemannian metrics on $T M$ for which horizontal and vertical distributions are mutually orthogonal. We use this condition to introduce the following definition.

Definition 2.1. A Riemannian $g$-natural metric $G$ on $T M$ is said to be of KaluzaKlein type if and only if horizontal and vertical distributions are $G$-orthogonal, that is, $\alpha_{2}=\beta_{2}=0$ in (2.1).

Next, the tangent sphere bundle of radius $r>0$ over a Riemannian manifold $(M, g)$ is the hypersurface

$$
T_{r} M=\left\{(x, u) \in T M: g_{x}(u, u)=r^{2}\right\} .
$$

The tangent space of $T_{r} M$, at a point $(x, u) \in T_{r} M$, is given by

$$
\left(T_{r} M\right)_{(x, u)}=\left\{X^{h}+Y^{v}: X \in M_{x}, Y \in\{u\}^{\perp} \subset M_{x}\right\} .
$$

When $r=1, T_{1} M$ is called the unit tangent (sphere) bundle.

By definition, $g$-natural metrics on $T_{1} M$ are the restrictions of $g$-natural metrics of $T M$ to its hypersurface $T_{1} M$. As proved in [5], every Riemannian $g$-natural metric $\tilde{G}$ on $T_{1} M$ is necessarily induced by a Riemannian $g$-natural $G$ on $T M$ of the special form

$$
\begin{aligned}
& G_{(x, u)}\left(X^{h}, Y^{h}\right)=(a+c) g_{x}(X, Y)+\beta g_{x}(X, u) g_{x}(Y, u), \\
& G_{(x, u)}\left(X^{h}, Y^{v}\right)=G_{(x, u)}\left(X^{v}, Y^{h}\right)=b g_{x}(X, Y), \\
& G_{(x, u)}\left(X^{v}, Y^{v}\right)=a g_{x}(X, Y),
\end{aligned}
$$

for three real constants $a, b, c$ and a smooth function $\beta:[0, \infty) \rightarrow \mathbb{R}$. Such a metric $\tilde{G}$ on $T_{1} M$ only depends on the value $d:=\beta(1)$ of $\beta$ at 1 . In particular, $\tilde{G}$ is Riemannian if and only if

$$
a>0, \quad \alpha:=a(a+c)-b^{2}>0 \quad \text { and } \quad \phi:=a(a+c+d)-b^{2}>0 .
$$

Returning to an arbitrary Riemannian $g$-natural metric on $T_{1} M$, a simple calculation, using Schmidt's orthonormalization process, shows that the vector field on $T M$ defined by

$$
N_{(x, u)}^{G}=\frac{1}{\sqrt{(a+c+d) \phi}}\left[-b u^{h}+(a+c+d) u^{v}\right],
$$

for all $(x, u) \in T M$, is unit normal at any point of $T_{1} M$.

We now define the tangential lift $X^{t_{G}}$ —with respect to $G$ - of a vector $X \in M_{x}$ to $(x, u) \in T_{1} M$ as the tangential projection of the vertical lift of $X$ to $(x, u)$ with respect to $N^{G}$, that is,

$$
X^{t_{G}}=X^{v}-G_{(x, u)}\left(X^{v}, N_{(x, u)}^{G}\right) N_{(x, u)}^{G}=X^{v}-\sqrt{\frac{\phi}{a+c+d}} g_{x}(X, u) N_{(x, u)}^{G} .
$$

If $X \in M_{x}$ is orthogonal to $u$, then $X^{t_{G}}=X^{v}$. 
The tangent space $\left(T_{1} M\right)_{(x, u)}$ of $T_{1} M$ at $(x, u)$ is spanned by vectors of the form $X^{h}$ and $Y^{t_{G}}$, where $X, Y \in M_{x}$. Using this fact, the Riemannian metric $\tilde{G}$ on $T_{1} M$, induced from $G$, is completely determined by the formulae

$$
\begin{aligned}
\tilde{G}_{(x, u)}\left(X^{h}, Y^{h}\right) & =(a+c) g_{x}(X, Y)+d g_{x}(X, u) g_{x}(Y, u), \\
\tilde{G}_{(x, u)}\left(X^{h}, Y^{t_{G}}\right) & =b g_{x}(X, Y), \\
\tilde{G}_{(x, u)}\left(X^{t_{G}}, Y^{t_{G}}\right) & =a g_{x}(X, Y)-\frac{\phi}{a+c+d} g_{x}(X, u) g_{x}(Y, u),
\end{aligned}
$$

for all $(x, u) \in T_{1} M$ and $X, Y \in M_{x}$. It should be noted that, by (2.11), the condition $b=0$ acquires a clear geometrical meaning. In fact, this condition is satisfied if and only if horizontal and vertical lifts are orthogonal with respect to $\tilde{G}$. Moreover, the condition $b=0$ characterizes metrics on $T_{1} M$ induced by Riemannian $g$-natural metrics on $T M$ of Kaluza-Klein type (Definition 2.1). For this reason, a Riemannian $g$-natural metric $\tilde{G}$ on $T_{1} M$ will be said to be of Kaluza-Klein type if horizontal and tangential distributions are $\tilde{G}$-orthogonal, that is, $b=0$ in (2.11).

It must be noted that the Sasaki metric on $T_{1} M$ is the Riemannian $g$-natural metric of Kaluza-Klein type of the form (2.11) with $a=1$ and $b=c=d=0$. Moreover, Kaluza-Klein metrics on the tangent bundle $T M$ are $g$-natural metrics satisfying (2.5) (see [26]), which induce on $T_{1} M$ the special subclass of Riemannian $g$-natural metrics of Kaluza-Klein type for which $b=d=0$.

\section{3. g-natural contact metric structures on $T_{1} M$}

A contact structure over a $(2 n-1)$-dimensional manifold $\bar{M}$ is a triplet $(\eta, \varphi, \xi)$, where $\eta$ is a global 1 -form on $\bar{M}$ (the contact form) satisfying $\eta \wedge(d \eta)^{n-1} \neq 0$ everywhere, $\xi$ is a global vector field (the characteristic vector field) and $\varphi$ is a global tensor of type $(1,1)$, such that

$$
\eta(\xi)=1, \quad \varphi(\xi)=0, \quad \eta \varphi=0, \quad \varphi^{2}=-I+\eta \otimes \xi .
$$

A Riemannian metric $g$ is said to be associated with the contact structure $(\eta, \varphi, \xi)$, if it satisfies

$$
\eta=g(\xi, \cdot), \quad d \eta=g(\cdot, \varphi \cdot), \quad g(\cdot, \varphi \cdot)=-g(\varphi \cdot, \cdot) .
$$

We refer to $(\bar{M}, \eta, g)$ or to $(\bar{M}, \eta, g, \xi, \varphi)$ as a contact metric manifold.

In [1], the first author and Abbassi investigated the conditions under which a Riemannian $g$-natural metric on $T_{1} M$ may be seen as a Riemannian metric associated with a very 'natural' contact structure. In fact, let $\tilde{G}$ be an arbitrary Riemannian $g$-natural metric over $T_{1} M$. We have already remarked that $N_{(x, u)}^{G}$, given by (2.9), is a unit vector field on $T M$, normal to $T_{1} M$ at any point. The tangent space to $T_{1} M$ at $(x, u)$ is given by

$$
\left(T_{1} M\right)_{(x, u)}=\operatorname{Span}(\tilde{\xi}) \oplus\left\{X^{h}: X \perp u\right\} \oplus\left\{X^{t_{G}}: X \perp u\right\},
$$

where $\tilde{\xi}$ is a vector field collinear to the geodesic flow, that is,

$$
\tilde{\xi}_{(x, u)}=r u^{h},
$$


$r$ being a positive constant. Hence, we consider the triple $(\tilde{\eta}, \tilde{\varphi}, \tilde{\xi})$, where $\tilde{\xi}$ is defined as in (3.5), $\tilde{\eta}$ is the 1 -form dual to $\tilde{\xi}$ through $\tilde{G}$, and $\tilde{\varphi}$ is completely determined by the relation

$$
\tilde{G}(Z, \tilde{\varphi} W)=(d \tilde{\eta})(Z, W),
$$

for all $Z, W$ vector fields on $T_{1} M$. Then, simple calculations show that

$$
\begin{aligned}
\tilde{\eta}\left(X^{h}\right) & =\frac{1}{r} g(X, u), \\
\tilde{\eta}\left(X^{t_{G}}\right) & =\operatorname{brg}(X, u),
\end{aligned}
$$

and

$$
\begin{aligned}
\tilde{\varphi}\left(X^{h}\right) & =\frac{1}{2 r \alpha}\left[-b X^{h}+(a+c) X^{t_{G}}+\frac{b d}{a+c+d} g(X, u) u^{h}\right], \\
\tilde{\varphi}\left(X^{t_{G}}\right) & =\frac{1}{2 r \alpha}\left[-a X^{h}+b X^{t_{G}}+\frac{\phi}{a+c+d} g(X, u) u^{h}\right],
\end{aligned}
$$

for all $X \in M_{x}$.

Since $u^{t_{G}}=(b /(a+c+d)) u^{h}$, it is easy to see that $\tilde{\eta}$ is well defined if and only if $b / r^{2}=b(a+c+d)$. When this condition holds, $\tilde{\eta}$ is homothetic, with homothety factor $r$, to the classical contact form on $T_{1} M$ (see, for example, [9] for a definition), and consequently, $\tilde{\eta}$ is again a contact form.

To ensure that $(\tilde{\eta}, \tilde{\varphi}, \tilde{\xi})$ is a contact structure, we must have $\tilde{\varphi}^{2}=-I+\tilde{\eta} \otimes \tilde{\xi}$. Hence, by (3.1) and (3.3) we get

$$
\frac{1}{r^{2}}=4 \alpha=a+c+d .
$$

Equation (3.4) may be used to express $d$ as a function of $a, b$ and $c$, and we obtain $d=(4 a-1)(a+c)-4 b^{2}$. In this way, we construct a family of contact metric structures $(\tilde{\eta}, \tilde{G}, \tilde{\varphi}, \tilde{\xi})$ over $T_{1} M$, depending on real parameters $a, b, c$ (satisfying some inequalities), to which we shall refer as g-natural contact metric structures on $T_{1} M$.

Note that, when $a=1 / 4$ and $b=c=d=0$ (and so, by (3.4), $r=2$ ), we get the standard contact metric structure of $T_{1} M$ (see, for example, [9, Ch. 9]). We also remark that $g$-natural contact metric structures associated with metrics of Kaluza-Klein type depend on two real parameters $a$ and $c$, as $b=0$ and, by (3.4), $d=(4 a-1)(a+c)$.

We recall that a contact metric manifold $(\bar{M}, \bar{\eta}, \bar{g})$ is said to be (locally) homogeneous contact if it admits a transitive (pseudo-)group of (local) isometries leaving invariant its contact form $\bar{\eta}$. We shall now prove the following theorem.

THEOREM 3.1. Let $(M, g)$ be a two-point homogeneous space. Then, any g-natural contact metric structure $(\tilde{\eta}, \tilde{G}, \tilde{\varphi}, \tilde{\xi})$ on $T_{1} M$ is homogeneous contact.

ProOF. Theorem 1.3 ensures that $\left(T_{1} M, \tilde{G}\right)$ is a homogeneous Riemannian manifold, for any Riemannian $g$-natural metric $\tilde{G}$. More precisely, it was proved in [19] that 
any (local) isometry $\psi$ of $(M, g)$ can be lifted to a (local) isometry $\Psi$ of $\left(T_{1} M, \tilde{G}\right)$, defined by

$$
\Psi(z)=\Psi(x, u)=\left(\psi(x), \psi_{*} u\right),
$$

for any unit tangent vector $z=(x, u) \in T_{1} M$.

Consider now an arbitrary $g$-natural contact metric structure $(\tilde{\eta}, \tilde{G}, \tilde{\varphi}, \tilde{\xi})$ on $T_{1} M$. Let $z=(x, u)$ be a point of $T_{1} M$ and $\gamma$ be the unique geodesic of $(M, g)$ such that $\gamma(0)=x$ and $\dot{\gamma}(0)=u$. We know from (3.1) that the characteristic vector field $\tilde{\xi}$ is defined through the geodesic flow, as

$$
\tilde{\xi}_{z}=r u^{h}=r \dot{\tilde{\gamma}}(0),
$$

where we put $\tilde{\gamma}(t):=(\gamma(t), \dot{\gamma}(t))$. Hence,

$$
\Psi_{* z} \tilde{\xi}_{z}=r \Psi_{* z} \dot{\tilde{\gamma}}(0)=r(\Psi \circ \tilde{\gamma})(0)
$$

Since $\gamma$ and $\psi$ respectively are a geodesic and a local isometry of $(M, g)$, the curve $\alpha(t):=\psi(\gamma(t))$ is again a geodesic of $(M, g)$ and, by (3.5), the curve

$$
\tilde{\alpha}(t):=(\Psi \circ \tilde{\gamma})(t)=\left(\psi(\gamma(t)), \psi_{*} \dot{\gamma}(t)\right)
$$

satisfies

$$
\tilde{\alpha}(0)=\Psi(z), \quad \dot{\tilde{\alpha}}(0)=\frac{1}{r} \Psi_{* z} \tilde{\xi}_{z}
$$

Hence,

$$
\tilde{\xi}_{\psi(z)}=\Psi_{* z} \tilde{\xi}_{z}
$$

and so $\tilde{\xi}$ is invariant under the isometries of the form $\Psi$, which act transitively on $\left(T_{1} M, \tilde{G}\right)$. Since $\Psi$ leaves both $\tilde{G}$ and $\tilde{\xi}$ invariant, it follows at once from (3.2) that $\Psi$ leaves $\tilde{\eta}$ invariant, that is, $(\tilde{\eta}, \tilde{G})$ is a homogeneous contact metric structure.

REMARK 3.2. A local version of Theorem 3.1 holds as well: if $(M, g)$ is locally isometric to a two-point homogeneous space, then any $g$-natural contact metric structure $(\tilde{\eta}, \tilde{G}, \tilde{\varphi}, \tilde{\xi})$ is locally homogeneous contact.

It is worth emphasizing the fact that Theorem 3.1 provides a large class of examples of homogeneous contact metric structures in any odd dimension. In fact, starting from any two-point homogeneous space, $g$-natural contact metric structures on its unit tangent sphere bundle provide such a family of examples, depending on three arbitrary parameters.

\section{Characterizations of two-point homogeneous spaces}

In general, a very important role in describing the geometry of a contact metric manifold $(\bar{M}, \eta, g)$ is played by the tensor

$$
h=\frac{1}{2} \mathcal{L}_{\xi} \varphi,
$$


where $\mathcal{L}$ denotes the Lie derivative. It was proved in [1] that at any point $(x, u)$ of the contact metric manifold $\left(T_{1} M, \tilde{\eta}, \tilde{G}\right)$, the tensor $\tilde{h}=(1 / 2) \mathcal{L}_{\tilde{\xi}} \tilde{\varphi}$ is described as follows:

$$
\begin{aligned}
\tilde{h}\left(X^{h}\right)=\frac{1}{4 \alpha} & {\left[-(a+c)(X-g(X, u) u)^{h}+a\left(R_{u} X\right)^{h}-2 b\left(R_{u} X\right)^{t_{G}}\right], } \\
\tilde{h}\left(X^{t_{G}}\right)=\frac{1}{4 \alpha} & {\left[-2 b X^{h}+b\left(1+\frac{d}{a+c+d}\right)\right.} \\
& \left.\times g(X, u) u^{h}+(a+c) X^{t_{G}}-a\left(R_{u} X\right)^{t_{G}}\right],
\end{aligned}
$$

for all $X \in M_{X}$, where $R$ is the curvature tensor of $(M, g)$, taken with the sign convention $R(X, Y)=\left[\nabla_{X}, \nabla_{Y}\right]-\nabla_{[X, Y]}$, and $R_{u} X=R(X, u) u$ denotes the Jacobi operator associated with $u$. In particular, (4.1) easily implies the following proposition.

Proposition 4.1. Let $(\tilde{\eta}, \tilde{G})$ be an arbitrary g-natural contact metric structure on $T_{1} M$. Then the following properties are equivalent.

(i) The horizontal distribution of $T_{1} M$ is $\tilde{h}$-invariant.

(ii) The tangential distribution of $T_{1} M$ is $\tilde{h}$-invariant.

(iii) $\tilde{G}$ is of Kaluza-Klein type.

It is worth briefly recalling the relationship between two-point homogeneous and Osserman spaces. A Riemannian manifold $(M, g)$ is called globally Osserman if the eigenvalues of the Jacobi operator $R_{u}$ are independent of both the unit tangent vector $u \in M_{x}$ and the point $x \in M$. The well-known Osserman conjecture states that any globally Osserman manifold is locally isometric to a two-point homogeneous space. Chi [16] and Nikolayevsky [21, 22] proved the Osserman conjecture in any dimension $n \neq 16$.

We are now ready to prove the converse of Theorem 3.1 for $g$-natural contact metric structures of Kaluza-Klein type. In this way, we generalize [10, Theorem 11], proving the following theorem.

THEOREM 4.2. Let $(M, g)$ be a Riemannian manifold of dimension $n \neq 16$ and $(\tilde{\eta}, \tilde{G})$ an arbitrary g-natural contact metric structure on $T_{1} M$, whose associated metric $\tilde{G}$ is of Kaluza-Klein type. Then $\left(T_{1} M, \tilde{\eta}, \tilde{G}\right)$ is a (locally) homogeneous contact metric manifold if and only if $(M, g)$ is (locally isometric to) a two-point homogeneous space.

PROOF. The 'if' part follows at once from Theorem 3.1. To prove the 'only if' part, consider a point $(x, u) \in T_{1} M$. Because of the symmetries of the curvature tensor, the Jacobi operator $R_{u}$ is self-adjoint and therefore diagonalizable. Let $\lambda_{1}, \ldots, \lambda_{n-1}$ be the eigenvalues of the Jacobi operator $R_{u}$ on the orthogonal subspace $u^{\perp}$ of $u$ in $T_{x} M$, and $e_{1}, \ldots, e_{n-1}$ be the corresponding unit eigenvectors. 
Consider now the contact metric manifold $\left(T_{1} M, \tilde{\eta}, \tilde{G}\right)$. By (4.1), taking into account the equality $b=0$ and the orthogonality of $u$ and $e_{i}$, we easily get

$$
\tilde{h}\left(e_{i}^{h}\right)=\frac{a \lambda_{i}-(a+c)}{4 \alpha} e_{i}^{h}, \quad \tilde{h}\left(e_{i}^{t_{G}}\right)=-\frac{a \lambda_{i}-(a+c)}{4 \alpha} e_{i}^{t_{G}},
$$

for all indices $i=1, \ldots, n-1$. Thus, $\left\{\tilde{\xi}, e_{i}^{h}, e_{i}^{t_{G}}\right\}$ is a basis of eigenvectors for $\tilde{h}$. Since $\left(T_{1} M, \tilde{\eta}, \tilde{G}\right)$ is (locally) homogeneous contact, the eigenvalues of $\tilde{h}$ on $\xi^{\perp}$ are constant [10, Lemma 10]. Hence, (4.2) implies at once that $\lambda_{i}$ is constant for all $i=1, \ldots, n-1$. So, $(M, g)$ is globally Osserman and this implies that $(M, g)$ is two-point homogeneous in any dimension $n \neq 16$.

As we showed in the proof of Theorem 3.1, if $(M, g)$ is two-point homogeneous, then a $g$-natural contact metric structure $(\tilde{\eta}, \tilde{G})$ on its unit tangent sphere bundle $T_{1} M$ is homogeneous, because its characteristic vector field $\tilde{\xi}$ (equivalently, by (3.1), the geodesic flow $u^{h}$ ) is invariant. This allows us to restate Theorem 4.2 in the following way, which does not involve contact geometry.

TheOREM 4.3. Let $(M, g)$ be a Riemannian manifold of dimension $n \neq 16$ and $\tilde{G}$ be an arbitrary $g$-natural metric on $T_{1} M$ of Kaluza-Klein type. Then, $(M, g)$ is (locally isometric to) a two-point homogeneous space if and only if $\left(T_{1} M, \tilde{G}\right)$ is (locally) homogeneous and the geodesic flow is invariant under the (local) isometries acting transitively on $T_{1} M$.

\section{5. $g$-natural $\boldsymbol{H}$-contact metric structures}

A contact metric manifold $(\bar{M}, \eta, g)$ is said to be $H$-contact if its characteristic vector field $\xi$ is harmonic, that is, is a critical point for the energy functional restricted to the set of all unit tangent vector fields. The definition above was given by the second author in [24], where he also proved that a contact metric manifold is $H$-contact if and only if $\xi$ is a Ricci eigenvector. This basic characterization implies that the class of $H$-contact metric manifolds is very large and includes several interesting classes of contact metric manifolds, such as Sasakian, $K$-contact, strongly $\varphi$-symmetric and $(\kappa, \mu)$-spaces (see [24]). Three-dimensional $H$-contact manifolds were further studied in $[18,23]$.

If $(M, g)$ is two-point homogeneous, then the standard contact metric structure on $T_{1} M$ is $H$-contact [12]. The converse holds in several classes of Riemannian manifolds [12, 13]. However, if $(M, g)$ is an Einstein manifold, then the standard contact metric structure on $T_{1} M$ is $H$-contact if and only if $(M, g)$ is 2-stein, that is,

$$
\sum_{i, j=1}^{n}\left(R_{u i u j}\right)^{2}=\mu(x)|u|^{4},
$$

for all $x \in M$ and $u \in T_{x} M$, where $\left\{e_{i}\right\}$ is an orthonormal basis of $T_{x} M$. Consequently, there exist Riemannian manifolds that are not two-point homogeneous, although the standard contact metric structure on their unit tangent sphere bundles is $H$-contact [17]. 
Now let $(M, g)$ be an arbitrary Riemannian manifold and consider any $g$-natural contact metric structure $(\tilde{\eta}, \tilde{G})$ on $T_{1} M$, such that the associated metric $\tilde{G}$ is of Kaluza-Klein type. The curvature tensor $\tilde{R}$ of an arbitrary Riemannian $g$-natural metric $\tilde{G}$ on $T_{1} M$ was calculated in [2]. In particular, if $\tilde{G}$ is of Kaluza-Klein type, then $b=0$ and so:

$$
\begin{aligned}
& \tilde{R}\left(X^{h}, Y^{h}\right) Z^{h} \\
& =\left\{R(X, Y) Z+\frac{a^{2}}{4 \alpha}[R(R(Y, Z) u, u) X\right. \\
& -R(R(X, Z) u, u) Y-2 R(R(X, Y) u, u) Z] \\
& +\frac{a d}{4 \alpha}[g(Z, u) R(X, Y) u+g(Y, u) R(X, u) Z-g(X, u) R(Y, u) Z] \\
& +\frac{d^{2}}{4 \alpha} g(Z, u)[g(Y, u) X-g(X, u) Y] \\
& +\frac{d}{4 \alpha(a+c+d)}\left\{a^{2}[g(R(Y, Z) u, R(X, u) u)\right. \\
& -g(R(X, Z) u, R(Y, u) u)-2 g(R(X, Y) u, R(Z, u) u)] \\
& +\operatorname{ad}[g(X, u) g(R(Y, u) Z, u)-g(Y, u) g(R(X, u) Z, u)] \\
& -3 a(a+c) g(R(X, Y) Z, u) \\
& +(a+c) d[g(X, u) g(Y, Z)-g(Y, u) g(X, Z)]\} u\}^{h} \\
& +\frac{1}{2}\left\{\left(\nabla_{Z} R\right)(X, Y) u\right\}^{t_{G}} ; \\
& \tilde{R}\left(X^{h}, Y^{t_{G}}\right) Z^{h} \\
& =\left\{-\frac{a^{2}}{2 \alpha}\left(\nabla_{X} R\right)(Y, u) Z+\frac{a^{2} d}{2 \alpha(a+c+d)} g\left(\left(\nabla_{X} R\right)(Y, u) Z, u\right) u\right\}^{h} \\
& +\left\{\frac{a^{2}}{4 \alpha} R(X, R(Y, u) Z) u+\frac{1}{2} R(X, Z) Y+\frac{a d}{4 \alpha}\right. \\
& \times[g(X, u) R(Y, u) Z-g(Z, u) R(X, Y) u]-\frac{d}{4 \alpha(a+c+d)} \\
& \times\left[a^{2} g(R(Y, u) Z, u)+\alpha g(Y, Z)\right] R_{u} X \\
& +\frac{d}{4 a(a+c+d)}[\operatorname{ag}(R(Y, u) Z, u)+(2(a+c)+d) g(Y, Z)] X \\
& \left.-\frac{d(4(a+c)+d)}{4 \alpha} g(X, u) g(Z, u) Y+\frac{(a+c) d}{2 \alpha} g(X, Y) Z\right\}^{t_{G}} ; \\
& \tilde{R}\left(X^{t_{G}}, Y^{t_{G}}\right) Z^{t_{G}}=\{g(Y, Z) X-g(X, Z) Y\}^{t_{G}},
\end{aligned}
$$

for all $x \in M,(x, u) \in T_{1} M$ and $X, Y, Z \in M_{x}$. The operation of tangential lift from $M_{x}$ to $(x, u) \in T_{1} M$ is applied only to vectors of $M_{x}$ which are orthogonal to $u$. 
Consider again a point $x \in M$, a unit tangent vector $(x, u) \in T_{1} M$ and $\tilde{G}$ a Riemannian $g$-natural metric of Kaluza-Klein type. If $\left\{e_{0}=u, e_{1}, \ldots, e_{n-1}\right\}$ is an orthonormal basis of $T_{x} M$, then by (2.11) it easily follows that

$$
\left\{\frac{1}{\sqrt{a+c+d}} e_{0}^{h}, \frac{1}{\sqrt{a+c}} e_{1}^{h}, \ldots, \frac{1}{\sqrt{a+c}} e_{n-1}^{h}, \frac{1}{\sqrt{a}} e_{1}^{t_{G}}, \ldots, \frac{1}{\sqrt{a}} e_{n-1}^{t_{G}}\right\}
$$

is an orthonormal basis of the tangent space $T_{u} T_{1} M$. Using such a basis, from Equations (5.1), (5.2) and (5.3) above, one easily obtains (see also [6])

$$
\begin{gathered}
\tilde{\varrho}\left(X^{h}, Y^{h}\right)=\varrho(X, Y)-\frac{a}{2(a+c)} \sum_{i=1}^{n-1} g\left(R\left(u, e_{i}\right) X, R\left(u, e_{i}\right) Y\right) \\
+\frac{a d}{2(a+c)(a+c+d)} g(R(X, u) u, R(Y, u) u) \\
+\frac{d(d-2(a+c+d))}{2 a(a+c+d)} g(X, Y) \\
+\frac{d}{a}\left(n+\frac{d}{2}\left(\frac{n-1}{a+c}-\frac{1}{a+c+d}\right)\right) g(X, u) g(Y, u), \\
\tilde{\varrho}\left(X^{h}, Y^{t_{G}}\right)=\frac{a}{2(a+c)}\left[\left(\nabla_{u} \varrho\right)(X, Y)-\left(\nabla_{Y} \varrho\right)(u, X)\right] \\
\quad+\frac{a d}{(a+c)(a+c+d)} g\left(\left(\nabla_{u} R\right)(X, u) Y, u\right) .
\end{gathered}
$$

By (3.1), the characteristic vector field of $(\tilde{\eta}, \tilde{G})$ is $\tilde{\xi}=r u^{h}$. Moreover, by (3.2), the contact distribution $\operatorname{Ker} \tilde{\eta}$ is spanned by horizontal and tangential lifts of vectors $Y$ orthogonal to $u$. Hence, from (5.4) we easily get

$$
\begin{aligned}
\tilde{\varrho}\left(\tilde{\xi}, Y^{h}\right) & =r\left(\varrho(u, Y)-\frac{a}{2(a+c)} \sum_{i=1}^{n-1} g\left(R\left(u, e_{i}\right) u, R\left(u, e_{i}\right) Y\right)\right), \\
\tilde{\varrho}\left(\tilde{\xi}, Y^{t_{G}}\right) & =\frac{r a}{2(a+c)}\left[\left(\nabla_{u} \varrho\right)(u, Y)-\left(\nabla_{Y} \varrho\right)(u, u)\right],
\end{aligned}
$$

for all $Y^{h}, Y^{t_{G}}$ in the contact distribution, that is, lifts of a tangent vector $Y$ orthogonal to $u$.

Thus, $\left(T_{1} M, \tilde{\eta}, \tilde{G}\right)$ is $H$-contact (equivalently, $\tilde{\xi}$ is a Ricci eigenvector) if and only if

$$
\begin{aligned}
\varrho(u, Y) & =\frac{a}{2(a+c)} \sum_{i=1}^{n-1} g\left(R\left(u, e_{i}\right) u, R\left(u, e_{i}\right) Y\right), \\
\left(\nabla_{u} \varrho\right)(u, Y) & =\left(\nabla_{Y} \varrho\right)(u, u),
\end{aligned}
$$

for all $Y$ orthogonal to $u$. In the special case of the standard contact metric structure of $T_{1} M$, that is, the $g$-natural contact metric structure determined by $a=1 / 4$ and 
$b=c=d=0$, we get the well-known characterization

$$
\begin{aligned}
2 \varrho(u, Y) & =\sum_{i=1}^{n-1} g\left(R\left(u, e_{i}\right) u, R\left(u, e_{i}\right) Y\right), \\
\left(\nabla_{u} \varrho\right)(u, Y) & =\left(\nabla_{Y} \varrho\right)(u, u),
\end{aligned}
$$

(see [12, 13]). As proved in [12], the second equation in (5.6) (and so in (5.5)) is equivalent to requiring that the Ricci tensor $\varrho$ of $(M, g)$ is $\operatorname{Codazzi}$, that is, satisfies

$$
\left(\nabla_{X} \varrho\right)(Y, Z)=\left(\nabla_{Y} \varrho\right)(X, Z)
$$

for all tangent vectors $X, Y, Z$. Thus, we have proved the following characterization.

Proposition 5.1. Let $(\tilde{\eta}, \tilde{G})$ be a g-natural contact metric structure on $T_{1} M$ of Kaluza-Klein type. Then $\left(T_{1} M, \tilde{\eta}, \tilde{G}\right)$ is $H$-contact if and only if:

(i) the Ricci tensor $\varrho$ of $(M, g)$ is Codazzi; and

(ii) $\varrho(u, Y)=a /(2(a+c)) \sum_{i=1}^{n-1} g\left(R\left(u, e_{i}\right) u, R\left(u, e_{i}\right) Y\right)$, for any orthogonal tangent vectors $u$ and $Y$.

In particular, if $(M, g)$ is Einstein, $\left(T_{1} M, \tilde{\eta}, \tilde{G}\right)$ is $H$-contact if and only if

$$
\sum_{i=1}^{n-1} g\left(R\left(u, e_{i}\right) u, R\left(u, e_{i}\right) Y\right)=0,
$$

for any orthogonal tangent vectors $u$ and $Y$.

The main result of [17] states that if $(M, g)$ is an Einstein manifold, then the standard contact metric structure on $T_{1} M$ is $H$-contact if and only if $(M, g)$ is 2-stein. Using Proposition 5.1, we now easily extend this result to the two-parameter family of contact metric structures defined by metrics of Kaluza-Klein type.

THEOREM 5.2. If $(M, g)$ is an Einstein manifold and $\tilde{G}$ is a Riemannian g-natural metric on $T_{1} M$ of Kaluza-Klein type, then $\left(T_{1} M, \tilde{\eta}, \tilde{G}\right)$ is $H$-contact if and only if $(M, g)$ is 2-stein.

We now prove a result related to Question 1.2. Specifically, we completely characterize 2-stein spaces in terms of $H$-contact metric structures on $T_{1} M$ defined by metrics of Kaluza-Klein type.

THEOREM 5.3. A Riemannian manifold $(M, g)$ is 2-stein if and only if there exist two Riemannian g-natural metrics of Kaluza-Klein type $\tilde{G}$ and $\tilde{G}^{\prime}$ on $T_{1} M$, satisfying $a c^{\prime} \neq a^{\prime} c$, such that the corresponding $g$-natural contact metric structures are $H$-contact.

In this case, all g-natural contact metric structures on $T_{1} M$, determined by g-natural metrics of Kaluza-Klein type, are H-contact.

PROOF. If $(M, g)$ is 2-stein, then it is Einstein, and so its Ricci tensor is parallel (in particular, is a Codazzi tensor). Moreover, condition (ii) in Proposition 5.1 is satisfied 
for any values of the parameters $a, c$. In fact, if $u, Y$ are orthogonal tangent vectors, then $\varrho(u, Y)=0$ because $(M, g)$ is Einstein, and $\sum_{i=1}^{n-1} g\left(R\left(u, e_{i}\right) u, R\left(u, e_{i}\right) Y\right)=0$ as proved in [17]. Hence, when $(M, g)$ is 2-stein, by Proposition 5.1 all $g$-natural contact metrics on $T_{1} M$, determined by a $g$-natural metric $\tilde{G}$ with $b=0$, are $H$-contact.

Conversely, suppose now that there exist two $g$-natural $H$-contact metric structures on $T_{1} M$, determined by two Riemannian $g$-natural metrics $\tilde{G}$ and $\tilde{G}^{\prime}$ with $b=b^{\prime}=0$. Fix two orthogonal tangent vectors $u$ and $Y$. Applying condition (ii) of Proposition 5.1, we obtain the system

$$
\begin{aligned}
& a\left(2 \varrho(u, Y)-\sum_{i=1}^{n-1} g\left(R\left(u, e_{i}\right) u, R\left(u, e_{i}\right) Y\right)\right)+2 c \varrho(u, Y)=0 \\
& a^{\prime}\left(2 \varrho(u, Y)-\sum_{i=1}^{n-1} g\left(R\left(u, e_{i}\right) u, R\left(u, e_{i}\right) Y\right)\right)+2 c^{\prime} \varrho(u, Y)=0,
\end{aligned}
$$

which, since $a c^{\prime} \neq a^{\prime} c$, necessarily implies that

$$
\begin{aligned}
\varrho(u, Y) & =0, \\
2 \varrho(u, Y) & =\sum_{i=1}^{n-1} g\left(R\left(u, e_{i}\right) u, R\left(u, e_{i}\right) Y\right) .
\end{aligned}
$$

The first equation in (5.7) easily yields that $(M, g)$ is Einstein. In fact, for any real number $\theta$, tangent vectors $\cos \theta u+\sin \theta Y,-\sin \theta u+\cos \theta Y$ are orthogonal. Hence,

$$
0=\varrho(\cos \theta u+\sin \theta Y,-\sin \theta u+\cos \theta Y)=\sin \theta \cos \theta(\varrho(u, u)-\varrho(Y, Y)),
$$

for any value of $\theta$, that is, $\varrho(u, u)=\varrho(Y, Y)$ for all orthogonal vectors $u, Y$. Moreover, $\varrho(u, Y)=0$. So $(M, g)$ is Einstein. The second equation in (5.7) then reduces to

$$
\sum_{i=1}^{n-1} g\left(R\left(u, e_{i}\right) u, R\left(u, e_{i}\right) Y\right)=0,
$$

which, as shown in [17], implies that the Einstein manifold $(M, g)$ is 2-stein. This completes the proof.

We remark that Theorem 5.3 ensures the existence of a large class of nonisometric $H$-contact metric structures on the unit tangent sphere bundle of any 2-stein space. For the list of 2-stein symmetric spaces, we refer to $[14,17]$.

\section{References}

[1] M. T. K. Abbassi and G. Calvaruso, ' $g$-natural contact metrics on unit tangent sphere bundles', Monatsh. Math. 151 (2006), 89-109.

[2] M. T. K. Abbassi and G. Calvaruso, 'The curvature tensor of $g$-natural metrics on unit tangent sphere bundles', Int. J. Contemp. Math. Sci. 3(6) (2008), 245-258.

[3] M. T. K. Abbassi and G. Calvaruso, 'Curvature properties of $g$-natural contact metric structures on unit tangent sphere bundles’, Beiträge Algebra Geom. 50(1) (2009), 155-178. 
[4] M. T. K. Abbassi, G. Calvaruso and D. Perrone, 'Harmonic maps defined by the geodesic flow', Houston J. Math. 36(1) (2010), 69-90.

[5] M. T. K. Abbassi and O. Kowalski, 'Naturality of homogeneous metrics on Stiefel manifolds $S O(m+1) / S O(m-1)$ ', Differential Geom. Appl. 28 (2010), 131-139.

[6] M. T. K. Abbassi and O. Kowalski, 'On Einstein Riemannian $g$-natural metrics on unit tangent sphere bundles', submitted.

[7] M. T. K. Abbassi and M. Sarih, 'On some hereditary properties of Riemannian $g$-natural metrics on tangent bundles of Riemannian manifolds', Differential Geom. Appl. 22(1) (2005), 19-47.

[8] M. Benyounes, E. Loubeau and C. M. Wood, 'Harmonic maps and Kaluza-Klein metrics on spheres', Arxiv:0809.2725v1.

[9] D. E. Blair, Riemannian Geometry of Contact and Symplectic Manifolds, Progress in Mathematics, 203 (Birkhäuser, Basel, 2002).

[10] E. Boeckx, D. Perrone and L. Vanhecke, 'Unit tangent sphere bundles and two-point homogeneous spaces', Period. Math. Hungar. 36 (1998), 79-95.

[11] E. Boeckx and L. Vanhecke, 'Geometry of the unit tangent sphere bundle' Public. Dep.to de Geometria y Topologia, Univ. Santiago de Compostela (Spain), 89 (1998), 5-17.

[12] E. Boeckx and L. Vanhecke, 'Harmonic and minimal vector fields on tangent and unit tangent bundles', Differential Geom. Appl. 13 (2000), 77-93.

[13] G. Calvaruso and D. Perrone, ' $H$-contact unit tangent sphere bundles', Rocky Mountain $J$. Math. 37(5) (2007), 1419-1442.

[14] P. Carpenter, A. Gray and T. J. Willmore, 'The curvature of Einstein symmetric spaces', Q. J. Math. 33 (1982), 45-64.

[15] J. Cheeger and D. Gromoll, 'On the structure of complete manifolds of nonnegative curvature', Ann. of Math. (2) 96 (1972), 413-443.

[16] Q. S. Chi, 'A curvature characterization of certain locally rank-one symmetric spaces', J. Differential Geom. 28 (1988), 187-202.

[17] S. H. Chun, J. H. Park and K. Sekigawa, ' $H$-contact unit tangent sphere bundles of Einstein manifolds', Q. J. Math., to appear. DOI: 10.1093/qmath/hap025.

[18] T. Koufogiorgos, M. Markellos and V. J. Papantoniou, 'The harmonicity of the Reeb vector field on contact metric three-manifolds', Pacific J. Math. 234(2) (2008), 325-344.

[19] O. Kowalski and M. Sekizawa, 'Invariance of $g$-natural metrics on tangent bundles', Differential Geom. Appl., 171-181 (World Sci. Publ., Hackensack, NJ, 2008).

[20] E. Musso and F. Tricerri, 'Riemannian metrics on tangent bundles', Ann. Mat. Pura Appl. 150(4) (1988), 1-20.

[21] Y. Nikolayevsky, 'Osserman manifolds of dimension 8', Manuscripta Math. 115 (2004), 31-53.

[22] Y. Nikolayevsky, 'Osserman conjecture in dimension $n \neq 8,16$ ', Math. Ann. 331 (2005), 505-522.

[23] D. Perrone, 'Harmonic characteristic vector fields on contact metric three manifolds', Bull. Aust. Math. Soc. 67 (2003), 305-315.

[24] D. Perrone, 'Contact metric manifolds whose characteristic vector field is a harmonic vector field', Differential Geom. Appl. 20 (2004), 367-378.

[25] J. A. Wolf, Spaces of Constant Curvature (McGraw-Hill, New York, 1967).

[26] C. M. Wood, 'An existence theorem for harmonic sections', Manuscripta Math. 68 (1990), 69-75.

G. CALVARUSO, Dipartimento di Matematica 'E. De Giorgi', Università del Salento, 73100 Lecce, Italy

e-mail: giovanni.calvaruso@unisalento.it

D. PERRONE, Dipartimento di Matematica 'E. De Giorgi', Università del Salento, 73100 Lecce, Italy

e-mail: domenico.perrone@unisalento.it 